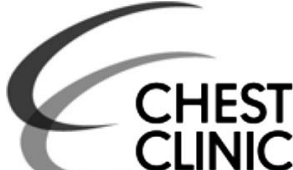

\section{Correspondence to}

Dr Kyle T S Pattinson, Nuffield Department of Clinical Neurosciences, University of Oxford, John Radcliffe Hospital, Oxford OX3 9DU, UK; Kyle.pattinson@nda.ox.ac. uk

Received 12 December 2014 Accepted 22 December 2014 Published Online First

14 January 2015

\title{
Functional brain imaging in respiratory medicine
}

Kyle Pattinson

\begin{abstract}
Discordance of clinical symptoms with markers of disease severity remains a conundrum in a variety of respiratory conditions. The breathlessness of chronic lung disease correlates poorly with spirometry, yet is a better predictor of mortality. In chronic cough, symptoms are often evident without clear physical cause. In asthma, the terms 'over perceivers' and 'under perceivers' are common parlance. In all these examples, aberrant brain mechanisms may explain the mismatch between symptoms and pathology. Functional MRI is a non-invasive method of measuring brain function. It has recently become significantly advanced enough to be useful in clinical research and to address these potential mechanisms. This article explains how FMRI works, current understanding from FMRI in breathlessness, cough and asthma and suggests possibilities for future research.
\end{abstract}

Over the past 20 years, functional MRI (FMRI) has transformed our understanding of the human brain. It has received much attention in both the scientific and popular press because the images generated provide a visually compelling means of explaining brain function. FMRI has elucidated a wide range of brain processes, from the underpinnings of complex human psychology to the mechanisms underlying autonomic function. The purpose of this article is to explain FMRI and its application to respiratory medicine. This will enable readers to better understand the increasing numbers of research papers using the technique.

FMRI holds great promise for identifying disease mechanisms and testing new treatments in respiratory medicine, as the brain represents a relatively untapped target. Symptoms of chronic lung disease and chronic cough are often debilitating, out of proportion to measurable pathology and refractory to conventional treatments. Although there are currently few FMRI studies in respiratory disease, the technique is well established in other areas of medicine - for example, the understanding of pain. In pain, FMRI has successfully translated hypotheses generated in animal models to humans. ${ }^{1}$ Furthermore, the technique has demonstrated how higher cortical centres influence transmission in ascending sensory pathways ('descending inhibition') and how these become maladaptive in chronic pain, exacerbating symptoms. In respiratory medicine, the goal for FMRI is to disentangle the relative contributions of the underlying pathology from altered brain processing in symptom perception. $^{2}$

\section{HOW DOES FMRI WORK AND WHAT ARE ITS LIMITATIONS?}

FMRI measures localised increases in blood oxyhaemoglobin and cerebral blood flow that occur as a consequence of brain activity. Because of the different magnetic properties of oxyhaemoglobin and deoxyhaemoglobin, brain activity increases the MR signal in areas of neural activation. This is known as the blood oxygenation level dependent (BOLD) response, is the source of image contrast in FMRI and has the great advantage of not requiring exogenous contrast agents, radiation or radioactive tracers.

A typical FMRI experiment involves intermittently administering an external stimulus (eg, a respiratory load) interspersed with rest periods while repeatedly acquiring MRI scans optimised for BOLD (figure 1). It is usual to simultaneously collect behavioural data (eg, breathlessness ratings) for incorporation into the analysis. FMRI analysis uses general linear modelling to detect correlations in the time course of the experimental stimulus (eg, an alternating respiratory load) with changes in BOLD in each image element (voxel) of the brain. The results from each individual are then merged and a statistical map of brain activity is created.

Unlike MRI scans used in clinical practice, the results of FMRI studies are usually obtained from experiments conducted in groups of $12-40$ participants. Although this number may seem low for those accustomed to reading research papers on clinical trials, FMRI obtains huge amounts of data from each individual. As long as a robust approach to analysis and interpretation is undertaken, FMRI can allow insights into brain function that would otherwise be impossible, making recruitment of large groups of subjects usually unnecessary.

Neurovascular coupling is the term used to describe the events that increase cerebral blood flow in concert with brain activity (figure 1). Anything that may affect neurovascular coupling may confound the interpretation of FMRI. Alterations in arterial carbon dioxide, $\mathrm{pH}$, arterial oxygen tension and intrathoracic pressure alter cerebral blood flow and thus BOLD. Drugs or disease states may directly affect chemical signalling mechanisms in astrocytes and cerebral blood vessels-for example, by changing vascular reactivity or cerebral metabolic rate. Ideally, such effects are either controlled-for example, by careful manipulation of end-tidal gas concentrations, or by the inclusion of control tasks-and/or accounted for in the statistical analysis. Correction for physiological noise (eg, respiratory and cardiac motion) and head movement is essential. Recent new approaches to FMRI data acquisition and analysis are much better equipped to deal with the issues of neurovascular coupling and physiological noise. This has tremendous implications on how FMRI will help in respiratory medicine.

As FMRI datasets comprise the time course of hundreds of thousands of individual voxels each 
Figure 1 Upper section: the pathway from neural activity to the haemodynamic response that is the basis of image contrast in functional MRI (FMRI). Drugs, disease and altered physiology may affect any point in this pathway, confounding interpretation of FMRI activations as neuronal in origin. Lower section: in its simplest form an alternating stimulus (eg, a flashing chequerboard alternating with rest periods for $15 \mathrm{~s}$ ) causes alternating on and off periods of electrical activity in certain brain regions. This electrical activity leads to a haemodynamic response. Multiple FMRI scans (each taking about $3 \mathrm{~s}$ ) capture the on and off periods. FMRI analysis regresses change in $\mathrm{T} 2$ * in each voxel (image element) against the time course of the alternating visual stimulus, and creates a statistical map of where changes in $\mathrm{T}^{*}$ correlate with the stimulus. This is interpreted as brain activity. BOLD, blood oxygenation level dependent.
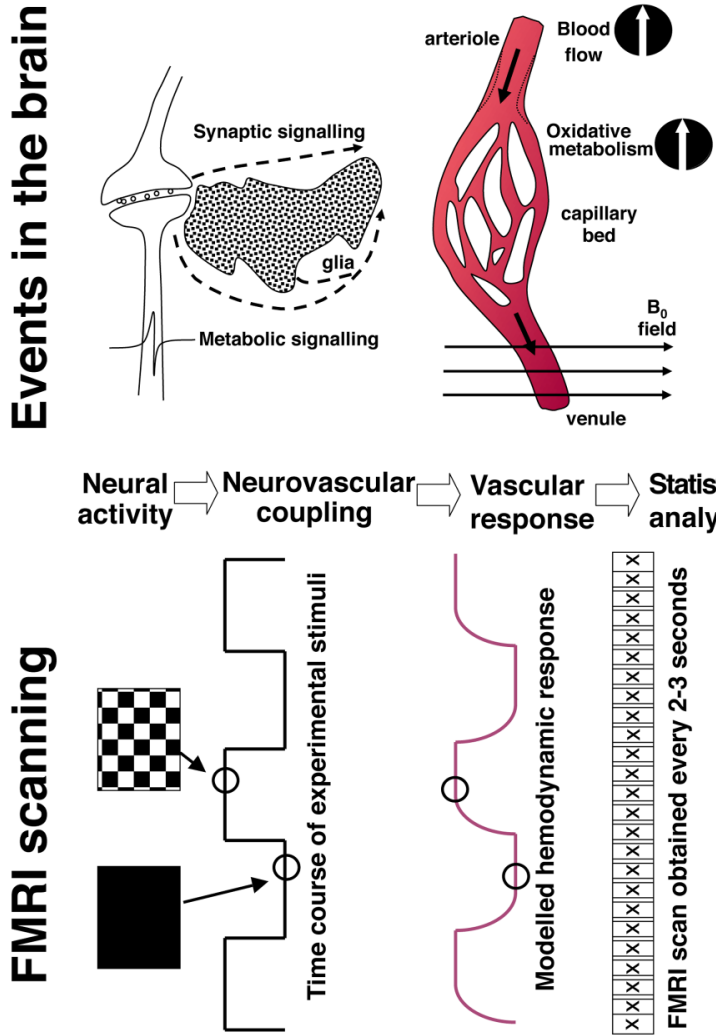

correlated with the stimulus of interest, appropriate multiple comparison correction is essential. An uncorrected $\mathrm{p}<0.0001$ may appear convincing, yet a typical FMRI dataset may compare 130000 voxels (MRI image elements) in which $\mathrm{p}<0.00000038$ would be equivalent to Bonferroni corrected $\mathrm{p}<0.05$. However, Bonferroni correction treats each voxel independently, whereas in reality brain activity is likely to be localised to groups of contiguous voxels (ie, a voxel is more likely to be 'active' if other 'active' voxels are nearby). This issue is addressed by the method of cluster-based thresholding, which takes into account both the multiple comparisons across the brain and correlated signal change in neighbouring voxels when determining significance.

An important limitation of FMRI lies not in the technique but in how the data are subsequently interpreted. Reverse inference is when authors reason 'backwards' that a brain activation represents a particular brain process based on the findings in different studies. An example may be that induced dyspnoea activates the insular cortex, and a previously published study of fear showed activation in the same area of the insular cortex. The reverse inference would be that this insular activation therefore represents fear. The reason that this is an issue is that FMRI cannot distinguish between different but co-localised neuronal populations which may subserve many different functions. Furthermore, FMRI cannot on its own tell whether a particular brain area is essential for a particular function.

\section{FMRI IN RESPIRATORY DISEASE: THE STORY SO FAR Breathlessness}

So far, fewer than 10 FMRI studies have been published on breathlessness, all investigating healthy volunteers. ${ }^{2}$ Activation patterns resemble those seen in FMRI studies of acute pain, implicating the insular and cingulate cortices as brain areas responsible for breathlessness processing. Variability in methods and low numbers of experimental subjects ( $\leq 9$ subjects in six of the 10 papers) highlight the need for more definitive studies.

\section{Cough}

FMRI studies using inhaled capsaicin to model the airway sensitisation of chronic cough demonstrate engagement of the insula and anterior cingulate cortices, known to modulate descending inhibitory pathways. ${ }^{3}$ Of particular interest is the involvement of the midbrain periaqueductal grey matter, a key nucleus that coordinates conscious with autonomic respiratory functions. Current findings suggest that heightened cough sensitivity is associated with abnormal function in a whole-brain network rather than a simple brainstem reflex circuit.

\section{Asthma}

The brain mechanisms of asthma have been explored in only four FMRI studies, each comparing patients with asthma with healthy controls. The most consistent finding is that the insular cortex seems to play a role in aberrant symptom perception.

\section{HOW MAY FMRI HELP?}

For FMRI to realise its potential in understanding respiratory disease, future work must investigate patients. Healthy volunteer models allow hypothesis testing without the confounds of disease state or drugs, but they are unable to address the conditioning, plasticity and long-term changes that are associated with chronic disease. For example, in patients with chronic pain, aberrant processing in the medial prefrontal cortex predicts transition to, and maintenance of, the chronic state.

\section{Breathlessness}

In chronic obstructive pulmonary disease it is well established that breathlessness correlates poorly with spirometry, yet is a better predictor of mortality. Pulmonary rehabilitation is one of 
the most effective treatments for breathlessness, yet it has no effect on spirometry and its benefits on breathlessness cannot be fully accounted for by improvements in respiratory muscle function or fitness. FMRI-derived brain biomarkers that predict breathlessness improvement in pulmonary rehabilitation would be obvious neuronal targets for new therapies that could be tested in patients.

\section{Chronic cough}

Patients with chronic cough demonstrate hypersensitivity of their airways ${ }^{3}$ leading to a disturbing increase in cough frequency, often without identifiable pathology. Although traditional models of cough focus on a reflex circuit in the brainstem, it is possible to voluntarily suppress or generate coughs. Although extremely challenging, the next key step is to determine how mechanisms discovered in volunteers translate to patients, and how established centrally acting drug treatments such as gabapentin affect descending inhibitory pathways in these patients.

\section{Asthma}

Discordance between asthma symptoms, airway inflammation and physiology is a recognised problem in many subjects with asthma, ${ }^{4}$ who often complain that their symptoms are not believed by medical professionals-for example, when spirometry is normal despite distressing symptoms. Exacerbation of asthma by stress and anxiety is well reported ${ }^{5}$ and may be due to maladaptive cortical influences on descending inhibition on the nerves that control airway calibre. Future studies need to take a whole-brain approach to determine network effects.

\section{CONCLUSIONS AND FUTURE DIRECTIONS}

FMRI offers a non-invasive safe opportunity to better understand human brain function in vivo. Although imaging studies of respiratory disease present challenges in experimental design and interpretation, the last few years have seen a much improved understanding of how to deal with these challenges. FMRI has now become sufficiently mature to earn its place as a useful tool for better understanding the brain processes underlying breathlessness and chronic cough, and this will naturally lead to the discovery of methods to test new treatments targeting the brain, complementing current therapies and hopefully addressing the huge unmet need for symptom relief in these patients.

Acknowledgements The author wishes to thank Dr Matthew Rowland, Dr Surinder Birring and Dr Ben Ainsworth for their constructive reviews of earlier versions of this paper.

Funding The author's research is supported by the Dunhill Medical Trust [grant number: R333/0214], the JABBS Foundation and the National Institute for Health Research (NIHR) Oxford Biomedical Research Centre based at The Oxford University Hospitals Trust, Oxford.

Competing interests None declared.

Provenance and peer review Commissioned; internally peer reviewed.

\section{REFERENCES}

1 Tracey I. Can neuroimaging studies identify pain endophenotypes in humans? Nat Rev Neurol 2011;7:173-81.

2 Herigstad M, Hayen A, Wiech K, et al. Dyspnoea and the brain. Respir Med 2011;105:809-17.

3 Ando A, Farrell MJ, Mazzone SB. Cough-related neural processing in the brain: a roadmap for cough dysfunction? Neurosci Biobehav Rev 2014;47C:457-468.

4 Haldar P, Pavord ID, Shaw DE, et al. Cluster analysis and clinical asthma phenotypes. Am J Respir Crit Care Med 2008;178:218-24.

5 Liu LY, Coe CL, Swenson CA, et al. School examinations enhance airway inflammation to antigen challenge. Am J Respir Crit Care Med 2002;165:1062-7. 